\title{
Research Article \\ Putting Yukawa-Like Modified Gravity (MOG) on the Test in the Solar System
}

\begin{abstract}
Lorenzo Iorio
Istituto Nazionale di Fisica Nucleare (INFN)-Sezione di Pisa, 56127 Pisa, Italy

Correspondence should be addressed to Lorenzo Iorio, lorenzo.iorio@libero.it

Received 4 September 2008; Revised 13 October 2008; Accepted 10 November 2008

We deal with a Yukawa-like long-range modified model of gravity (MOG) which recently allowed to successfully accommodate many astrophysical and cosmological features without resorting to dark matter. On Solar System scales, MOG predicts anomalous retrograde secular precessions of the planetary longitudes of the perihelia $\omega$. Their existence has been put on the test here by taking the ratios of the observationally estimated Pitjeva's corrections to the standard Newtonian/Einsteinian perihelion precessions for different pairs of planets. It turns out that MOG, in the present form which turned out to be phenomenologically successful on astrophysical scales, is ruled out at more than $3 \sigma$ level in the Solar System. If and when other teams of astronomers will independently estimate their own corrections to the usual precessions of the perihelia, it will be possible to repeat such a test.
\end{abstract}

Copyright ( $) 2008$ Lorenzo Iorio. This is an open access article distributed under the Creative Commons Attribution License, which permits unrestricted use, distribution, and reproduction in any medium, provided the original work is properly cited.

\section{Introduction}

The modified gravity (MOG) theory put forth in [1] was used successfully to describe various observational phenomena on astrophysical and cosmological scales without resorting to dark matter (see [2] and references therein). It is a fully covariant theory of gravity which is based on the existence of a massive vector field coupled universally to matter. The theory yields a Yukawa-like modification of gravity with three constants which, in the most general case, are running; they are present in the theory's action as scalar fields which represent the gravitational constant, the vector field coupling constant, and the vector field mass. Actually, the issue of the running of the parameters of modified models of gravity is an old one, known in somewhat similar contexts since the early 1990s (see, e.g., [3] and references therein). An approximate solution of the MOG field equations [4] allows to compute their values as functions of the source's mass.

The resulting Yukawa-type modification of the inversesquare Newton's law in the gravitational field of a central mass $M$ is $[2,4]$

$$
A_{\mathrm{MOG}}=-\frac{G_{\mathrm{N}} M}{r^{2}}\{1+\alpha[1-(1+\mu r) \exp (-\mu r)]\},
$$

where $G_{\mathrm{N}}$ is the Newtonian gravitational constant and $[2,4]$

$$
\begin{gathered}
\alpha=\frac{M}{\left(\sqrt{M}+C_{1}^{\prime}\right)^{2}}\left(\frac{G_{\infty}}{G_{\mathrm{N}}}-1\right), \\
G_{\infty} \approx 20 G_{\mathrm{N}}, \quad C_{1}^{\prime} \approx 25000 \mathrm{M}_{\odot}^{1 / 2}, \\
\mu=\frac{C_{2}^{\prime}}{\sqrt{M}}, \quad C_{2}^{\prime} \approx 6250 \mathrm{M}_{\odot}^{1 / 2} \mathrm{kpc}^{-1} .
\end{gathered}
$$

Such values have been obtained in [4] as a result of the fit of the velocity rotation curves of some galaxies in the framework of the searches for an explanation of the flat rotation curves of galaxies without resorting to dark matter.

In this paper, we will put (1) on the test in the Solar System in order to check if it is compatible with the latest determinations of the corrections $\langle\Delta \dot{\omega}\rangle$ to the usual Newtonian/Einsteinian planetary perihelion precessions $[5,6]$. Such quantities are solved for parameters phenomenologically estimated in a least-square sense by fitting about one century of observations of various kinds concerning planetary motions with their values theoretically predicted by simultaneously integrating the equations of motions of the major bodies of the Solar System written in terms of a complete suite of dynamical force models. They encompass all the known Newtonian effects along with those predicted by general relativity, except for the Lense-Thirring 
effect [7-9] which was left unmodelled (see below). Thus, $\langle\Delta \dot{\omega}\rangle$ account for any unmodelled/mismodelled dynamical features of motion and are good, in principle, to put on the test MOG, provided that they are used in a suitable way.

Note that Moffat and Toth explicitly write in [2] that (1), with (2), is not in contradiction with the present-day knowledge of Solar System dynamics. We will show that it is not so, also, for any other (nonzero) values of $\alpha$ and $\mu$, with the only quite general condition that $\mu r \ll 1$ in Solar System, as it must be for any long-range modified model of gravity. It is interesting to point out that Yukawa-like modifications of Newton's law might also be tested in the context of stellar dynamics [10]. Now, we outline the procedure that we will follow.

Generally speaking, let long-range modified model of gravity (LRMOG) be a given exotic model of modified gravity parameterized in terms of, say, $K$, in such a way that $K=0$ would imply no modifications of gravity at all. Let $\mathcal{P}(\mathrm{LRMOG})$ be the prediction of a certain effect induced by such a model like, for example, the secular precession of the perihelion of a planet. For all the exotic models considered, it turns out that

$$
\mathcal{P}(\mathrm{LRMOG})=K g(a, e),
$$

where $g$ is a function of the system's orbital parameters $a$ (semimajor axis) and $e$ (eccentricity); such $g$ is a peculiar consequence of the model LRMOG (and of all other models of its class with the same spatial variability). (In our case, it will be $K=-\alpha \mu^{2}$, as we will see in Section 2). Now, let us take the ratio of $\mathcal{P}(\mathrm{LRMOG})$ for two different systems A and B, for example, two Solar System's planets: $\mathcal{P}_{\mathrm{A}}(\mathrm{LRMOG}) / \mathcal{P}_{\mathrm{B}}(\mathrm{LRMOG})=g_{\mathrm{A}} / g_{\mathrm{B}}$. The model's parameter $K$ has now been canceled, but we still have a prediction that retains a peculiar signature of that model, that is, $g_{\mathrm{A}} / g_{\mathrm{B}}$. Of course, such a prediction is valid if we assume $K$ is not zero, which is just the case both theoretically (if $K$ was zero, no modifications of gravity at all would occur) and observationally because $K$ is usually determined by other independent long-range astrophysical/cosmological observations. Otherwise, one would have the meaningless prediction $0 / 0$. The case $K=0$ (or $K \leq \bar{K}$ ) can be, instead, usually tested by taking one perihelion precession at a time. If we have observational determinations $\mathcal{O}$ for $\mathrm{A}$ and $\mathrm{B}$ of the effect considered above such that they are affected also by LRMOG (it is just the case for the purely phenomenologically estimated corrections to the standard Newton-Einstein perihelion precessions, since LRMOG has not been included in the dynamical force models of the ephemerides adjusted to the planetary data in the leastsquare parameters' estimation process by Pitjeva $[5,6])$, we can construct $\mathcal{O}_{\mathrm{A}} / \mathcal{O}_{\mathrm{B}}$ and compare it with the prediction for it by LRMOG, that is, with $g_{\mathrm{A}} / g_{\mathrm{B}}$. (If they are differential quantities constructed by contrasting observations to predictions obtained by analytical force models of canonical Newtonian/Einsteinian effects, $\mathcal{O}$ are, in principle, affected also by the mismodelling in them.) Note that $\delta \mathcal{O} / \mathcal{O}>1$ only means that $\mathcal{O}$ is compatible with zero, being possible a nonzero value smaller than $\delta \mathcal{O}$. Thus, it is perfectly meaningful to construct $\mathcal{O}_{A} / \mathcal{O}_{B}$. Its uncertainty will be conservatively evaluated as $\left|1 / \mathcal{O}_{\mathrm{B}}\right| \delta \mathcal{O}_{\mathrm{A}}+\left|\mathcal{O}_{\mathrm{A}} / \mathcal{O}_{\mathrm{B}}^{2}\right| \delta \mathcal{O}_{\mathrm{B}}$. As a result, $\mathcal{O}_{\mathrm{A}} / \mathcal{O}_{\mathrm{B}}$ will be compatible with zero. Now, the question is "is it the same for $g_{\mathrm{A}} / g_{\mathrm{B}}$ as well?" If yes, that is, if

$$
\frac{\mathcal{O}_{\mathrm{A}}}{\mathcal{O}_{\mathrm{B}}}=\frac{\mathcal{P}_{\mathrm{A}}(\text { LRMOG })}{\mathcal{P}_{\mathrm{B}}(\text { LRMOG })}
$$

within the errors, or, equivalently, if

$$
\left|\frac{\mathcal{O}_{\mathrm{A}}}{\mathcal{O}_{\mathrm{B}}}-\frac{\mathcal{P}_{\mathrm{A}}(\mathrm{LRMOG})}{\mathcal{P}_{\mathrm{B}}(\mathrm{LRMOG})}\right|=0
$$

within the errors, LRMOG survives (and the use of the single perihelion precessions can be used to put upper bounds on $K)$. Otherwise, LRMOG is challenged.

\section{The Predicted Perihelion Precessions and the Confrontation with the Estimated Nonstandard Rates}

In the case of the Sun, (2) yield

$$
\alpha_{\odot} \approx 3 \times 10^{-8}, \quad \mu \approx 3 \times 10^{-5} \mathrm{AU}^{-1},
$$

so that

$$
\alpha_{\odot} \mu^{2}=3 \times 10^{-17} \mathrm{AU}^{-2} .
$$

Since in the Solar System $\mu r \approx 10^{-5}-10^{-4}$, we can safely assume $\exp (-\mu r) \approx 1-\mu r$, so that

$$
A_{\mathrm{MOG}} \approx-\frac{G_{\mathrm{N}} M}{r^{2}}\left(1+\alpha \mu^{2} r^{2}\right) .
$$

As a result, a radial, uniform perturbing acceleration

$$
A=-G_{\mathrm{N}} M \alpha \mu^{2} \approx 10^{-19} \mathrm{~m} \mathrm{~s}^{-2}
$$

is induced.

The secular, that is, averaged over one orbital revolution, effect of a small radial and unform perturbing acceleration on the longitude of the perihelion of a planet $\omega$ has been perturbatively worked out, for example, by Sanders in [11]; it amounts to

$$
\begin{aligned}
\left\langle\frac{d \omega}{d t}\right\rangle & =A \sqrt{\frac{a\left(1-e^{2}\right)}{G_{\mathrm{N}} M}} \\
& =-\alpha \mu^{2} \sqrt{G_{\mathrm{N}} M a\left(1-e^{2}\right)} .
\end{aligned}
$$

Clearly, using only one perihelion rate at a time would yield no useful information on MOG due to the extreme smallness of the perturbing acceleration, as told to us by (9). Thus, let us take the ratios of the perihelion precessions. It must be noted that the following analysis is, in fact, truly independent of the values of $\alpha$ and $\mu$, provided only that $\alpha \mu^{2} r^{2} \ll 1$ in the Solar System so that the perturbative approach can be applied to (8); the condition $\mu r \ll 1$ is the cornerstone of any long-range modified models of gravity, and should 
TABLE 1: Inner planets. First row: estimated corrections to the standard precessions of the longitudes of the perihelia in $10^{-4} " \mathrm{cy}^{-1}\left({ }^{\prime \prime} \mathrm{cy}-1 \rightarrow\right.$ arcseconds per century), from [6, Table 3] (apart from Venus). The quoted errors, in $10^{-4}{ }^{\prime \prime} \mathrm{cy}^{-1}$, are not the formal ones but are realistic. The formal errors are quoted in square brackets (Pitjeva, personal communication to the author, November 2005). The units are 10 ${ }^{-4}$ " $\mathrm{cy}^{-1}$. Second row: semimajor axes, in astronomical units (AU). Their formal errors are in [5, Table 4], in m. Third row: eccentricities. Fourth row: orbital periods in years. The result for Venus has been recently obtained by including the Magellan radiometric data (Pitjeva, personal communication to the author, June 2008).

\begin{tabular}{lcccc}
\hline & Mercury & Venus & Earth & Mars \\
\hline$\langle\Delta \dot{\omega}\rangle\left(10^{-4 \prime} \mathrm{cy}^{-1}\right)$ & $-36 \pm 50[42]$ & $-4 \pm 5[1]$ & $-2 \pm 4[1]$ & $1 \pm 5[1]$ \\
$a(\mathrm{AU})$ & 0.387 & 0.723 & 1.000 & 1.523 \\
$e$ & 0.2056 & 0.0067 & 0.0167 & 0.0934 \\
$P(\mathrm{yr})$ & 0.24 & 0.61 & 1.00 & 1.88 \\
\hline
\end{tabular}

TABle 2: First two columns: pair of planets. Third column: $\Pi$ for that pair of planets. The errors come from the realistic uncertainties in $\langle\Delta \dot{\omega}\rangle$. Fourth column: $\mathcal{A}$ for that pair of planets. Fifth column: $\sigma$ level of discrepancy between $\Pi$ and $\mathcal{A}$ for that pair of planets.

\begin{tabular}{lcccc}
\hline A & B & $\Pi$ & $\mathcal{A}$ & $\sigma$ \\
\hline Venus & Mercury & $0.1 \pm 0.3$ & 1.4 & 4 \\
Earth & Mercury & $0.05 \pm 0.18$ & 1.64 & 8 \\
Mars & Mercury & $-0.03 \pm 0.18$ & 2.02 & 11 \\
\hline
\end{tabular}

$\alpha \approx 1$, the planetary orbits would have been distorted in a so huge manner that it would have been detected since long time. Applying the scheme outlined in Section 1 to our case in which $K=-\alpha \mu^{2}$ and $g(a, e)=\sqrt{G_{N} M a\left(1-e^{2}\right)}$, one can construct

$$
\Pi \equiv \frac{\left\langle\Delta \dot{\omega}_{\mathrm{A}}\right\rangle}{\left\langle\Delta \dot{\omega}_{\mathrm{B}}\right\rangle}
$$

with the estimated corrections $\langle\Delta \dot{\omega}\rangle$ to the standard Newtonian/Einsteinian perihelion precessions of planets $\mathrm{A}$ and $\mathrm{B}$, listed in Table 1, and compare them to the theoretical prediction

$$
\mathcal{A} \equiv \sqrt{\frac{a_{\mathrm{A}}\left(1-e_{\mathrm{A}}^{2}\right)}{a_{\mathrm{B}}\left(1-e_{\mathrm{B}}^{2}\right)}},
$$

obtained from (10), for that pair of planets A and B. The results are in Table $2 .|\Pi-\mathcal{A}|$ is different from zero at more than $3 \sigma$ level for $\mathrm{A}=$ Venus, $\mathrm{B}=$ Mercury, $\mathrm{A}=$ Earth, $\mathrm{B}=$ Mercury and $\mathrm{A}=$ Mars, $\mathrm{B}=$ Mercury. It is important to note that the errors have been conservatively evaluated as

$$
\delta \Pi \leq|\Pi|\left(\frac{\delta\left\langle\Delta \dot{\omega}_{\mathrm{A}}\right\rangle}{\left|\left\langle\Delta \dot{\omega}_{\mathrm{A}}\right\rangle\right|}+\frac{\delta\left\langle\Delta \dot{\omega}_{\mathrm{B}}\right\rangle}{\left|\left\langle\Delta \dot{\omega}_{\mathrm{B}}\right\rangle\right|}\right)
$$

because of the existing correlations among the estimated corrections to the precessions of perihelia. (The maximum correlation, 26\%, occurs for the Earth and Mercury (Pitjeva, personal communication to the author, November 2005)).

If we repeat our analysis by subtracting from $\langle\Delta \dot{\omega}\rangle$ the main canonical unmodelled effect, that is, the general relativistic Lense-Thirring precessions [7-9] induced by the
TABLE 3: General relativistic Lense-Thirring precessions of the longitudes of perihelia of the inner planets of the Solar System in $10^{-4 \prime \prime} \mathrm{cy}^{-1}$.

\begin{tabular}{lcccc}
\hline & Mercury & Venus & Earth & Mars \\
\hline$\dot{\bar{\omega}}^{(\mathrm{LT})}\left(10^{-4 \prime \prime} \mathrm{cy}^{-1}\right)$ & -20 & -3 & -1 & -0.3 \\
\hline
\end{tabular}

TABle 4: First two columns: pair of planets. Third column: $\Pi^{\star}$ for that pair of planets including the unmodelled general relativistic Lense-Thirring effect. The errors come from the realistic uncertainties in $\langle\Delta \dot{\omega}\rangle$. Fourth column: $\mathcal{A}$ for that pair of planets. Fifth column: $\sigma$ level of discrepancy between $\Pi$ and $\mathcal{A}$ for that pair of planets.

\begin{tabular}{lcccc}
\hline A & $\mathrm{B}$ & $\Pi^{\star}$ & $\mathcal{A}$ & $\sigma$ \\
\hline Venus & Mercury & $0.06 \pm 0.51$ & 1.4 & 2.7 \\
Earth & Mercury & $0.06 \pm 0.44$ & 1.64 & 3.5 \\
Mars & Mercury & $-0.08 \pm 0.56$ & 2.02 & 3.7 \\
\hline
\end{tabular}

Sun's angular momentum [12] shown in Table 3, that is, if we use

$$
\Pi^{\star} \equiv \frac{\left\langle\Delta \dot{\omega}_{\mathrm{A}}\right\rangle^{\star}}{\left\langle\Delta \dot{\omega}_{\mathrm{B}}\right\rangle^{\star}}=\frac{\left\langle\Delta \dot{\omega}_{\mathrm{A}}\right\rangle-\dot{\omega}_{\mathrm{A}}^{(\mathrm{LT})}}{\left\langle\Delta \dot{\omega}_{\mathrm{B}}\right\rangle-\dot{\omega}_{\mathrm{B}}^{(\mathrm{LT})}},
$$

the situation does not substantially change, apart from the sigma level at which $\left|\Pi^{\star}-\mathcal{A}\right|$ is not compatible with zero, as shown in Table 4.

The availability of the corrections to the usual rates of perihelia of several planets allows us to put on the test MOG also in another way as well. The acceleration law of (1) can also be recast in the commonly used Yukawa form [4]

$$
A_{\mathrm{Y}}=-\frac{G_{\mathrm{Y}} M}{r^{2}}\left[1+\alpha_{\mathrm{Y}}\left(1+\frac{r}{\lambda}\right) \exp \left(-\frac{r}{\lambda}\right)\right],
$$

where

$$
\begin{gathered}
G_{\mathrm{Y}}=\frac{G_{\mathrm{N}}}{1+\alpha_{\mathrm{Y}}}, \\
\alpha_{\mathrm{Y}}=-\frac{\left(G_{\infty}-G_{\mathrm{N}}\right) M}{\left(G_{\infty}-G_{\mathrm{N}}\right) M+G_{\mathrm{N}}\left(\sqrt{M}+C_{1}^{\prime}\right)^{2}}, \\
\lambda=\frac{1}{\mu} .
\end{gathered}
$$


In the case of the Sun,

$$
\begin{gathered}
\alpha_{\mathrm{Y}}^{\odot}=-3.04 \times 10^{-8}, \quad G_{\mathrm{Y}}=1.00000003040 G_{\mathrm{N}}, \\
\lambda=33000 \mathrm{AU} .
\end{gathered}
$$

A Yukawa-type acceleration of the form of (15) has been tested [13] in the Solar System without any a priori assumption on the size of $\alpha_{\mathrm{Y}}$; concerning $\lambda$, it was only assumed that $\lambda \gtrsim a e$. By using the corrections to the standard rates of the perihelia of $\mathrm{A}=$ Earth and $\mathrm{B}=$ Mercury quoted in Table 1, Iorio [13] found

$$
\lambda=\frac{a_{\mathrm{B}}-a_{\mathrm{A}}}{\ln \left(\sqrt{a_{\mathrm{B}} / a_{\mathrm{A}}} \Pi\right)}=0.182 \pm 0.183 \mathrm{AU}
$$

which contradicts (17). (The strength parameter $\alpha$ used in [13] can be identified with $\alpha_{\mathrm{Y}}$ here.) Using the data for Venus in the equation for $\alpha_{\mathrm{Y}}$ [13]

$$
\alpha_{\mathrm{Y}}=\frac{2 \lambda^{2}\langle\Delta \dot{\dot{\omega}}\rangle}{\sqrt{G_{\mathrm{Y}} M a}} \exp \left(\frac{a}{\lambda}\right)
$$

yields

$$
\alpha_{\mathrm{Y}}=(-1 \pm 4) \times 10^{-11},
$$

which is three orders of magnitude smaller than the result of (17). (According to (17), using $G_{\mathrm{N}}$ in (19) instead of $G_{Y}$, as done in [13], does not produce appreciable modifications of the results.)

If we use $\Pi^{\star}$ for the Earth and Mercury in (18) and $\langle\Delta \dot{\boldsymbol{\omega}}\rangle^{\star}$ for Venus in (19), the results do not change appreciably; indeed, we have

$$
\lambda=0.2 \pm 0.4 \mathrm{AU}, \quad \alpha_{\mathrm{Y}}=(-0.3 \pm 2.7) \times 10^{-11} .
$$

\section{Conclusions}

In the framework of the attempts of explaining certain astrophysical and cosmological features without invoking dark matter, MOG $[1,4]$ is a long-range modified model of gravity, based on a vector field and three scalar fields representing running constants, which assumes a Yukawalike form. Recent developments of this theory allowed their proponents to fix $[2,4]$ the values of the constants entering it. We have shown that, on Solar System scales, MOG yields an anomalous uniform acceleration inducing retrograde planetary perihelion precessions. We put on the test the possibility that such exotic precessions exist by comparing the ratio of them $\mathcal{A}$ for different pairs of planets to the ratio $\Pi$ of the corrections to the usual Newtonian/Einsteinian precessions estimated by Pitjeva which account for any unmodelled/mismodeleld dynamical effects. It turns out that $\Pi \neq \mathcal{A}$ at more than $3 \sigma$ level even by including in $\Pi$ the main unmodelled canonical effect, that is, the general relativistic Lense-Thirring precessions. Conversely, using the estimated corrections to the planetary perihelion rates to phenomenologically determine the strength parameter of the putative MOG Yukawa force and its range yields values which are neatly incompatible with those of MOG $[2,4]$. In assessing the results presented here, it must be considered that, at present, no other researchers have estimated the nonstandard part of the planetary perihelion motions; it would certainly be useful to repeat the present analysis if and when other teams of astronomers will estimate their own set of corrections to the standard perihelion precessions as well.

\section{References}

[1] J. W. Moffat, "Scalar-tensor-vector gravity theory," Journal of Cosmology and Astroparticle Physics, vol. 2006, no. 3, p. 004, 2006.

[2] J. W. Moffat and V. T. Toth, "Testing modified gravity with globular cluster velocity dispersions," Astrophysical Journal, vol. 680, no. 2, pp. 1158-1161, 2008.

[3] O. Bertolami, J. M. Mourão, and J. Pérez-Mercader, "Quantum gravity and the large scale structure of the universe," Physics Letters B, vol. 311, no. 1-4, pp. 27-33, 1993.

[4] J. W. Moffat and V. T. Toth, "Fundamental parameterfree solutions in modified gravity," http://arxiv.org/abs/ 0712.1796v4.

[5] E. V. Pitjeva, "High-precision ephemerides of planets-EPM and determination of some astronomical constants," Solar System Research, vol. 39, no. 3, pp. 176-186, 2005.

[6] E. V. Pitjeva, "Relativistic effects and solar oblateness from radar observations of planets and spacecraft," Astronomy Letters, vol. 31, no. 5, pp. 340-349, 2005.

[7] J. Lense and H. Thirring, "Über den Einfluß der Eigenrotation der Zentralkorper auf die Bewegung der Planeten und Monde nach der Einsteinschen Gravitationstheorie," Physikalische Zeitschrift, vol. 18, pp. 156-163, 1918.

[8] B. Mashhoon, F. W. Hehl, and D. S. Theiss, "On the gravitational effects of rotating masses: the Thirring-Lense papers," General Relativity and Gravitation, vol. 16, no. 8, pp. 711-750, 1984.

[9] B. Mashhoon, F. W. Hehl, and D. S. Theiss, "On the gravitational effects of rotating masses: the Thirring-Lense papers," in Nonlinear Gravitodynamics, R. J. Ruffini and C. Sigismondi, Eds., pp. 349-388, World Scientific, Singapore, 2003.

[10] O. Bertolami and J. Páramos, "Astrophysical constraints on scalar field models," Physical Review D, vol. 71, no. 2, Article ID 023521, 10 pages, 2005.

[11] R. H. Sanders, "Solar system constraints on multifield theories of modified dynamics," Monthly Notices of the Royal Astronomical Society, vol. 370, no. 3, pp. 1519-1528, 2006.

[12] L. Iorio, "First preliminary tests of the general relativistic gravitomagnetic field of the Sun and new constraints on a Yukawa-like fifth force from planetary data," Planetary and Space Science, vol. 55, no. 10, pp. 1290-1298, 2007.

[13] L. Iorio, "Constraints on the range $\lambda$ of Yukawa-like modifications to the Newtonian inverse-square law of gravitation from Solar System planetary motions," Journal of High Energy Physics, vol. 2007, no. 10, p. 041, 2007. 